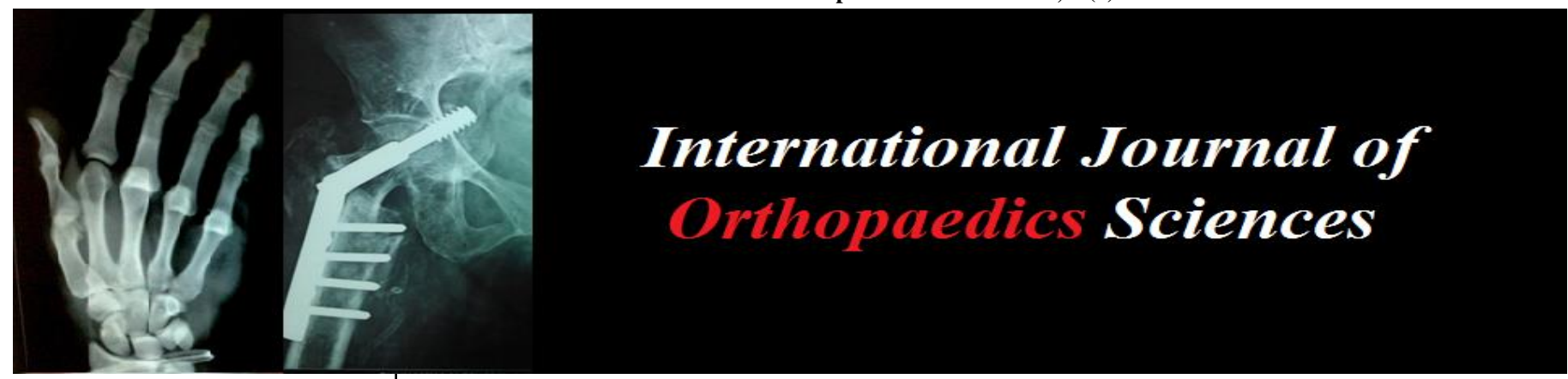

E-ISSN: 2395-1958

P-ISSN: 2706-6630

IJOS 2022; 8 (1): 318-322

(C) 2022 IJOS

www.orthopaper.com

Received: 06-10-2021

Accepted: 13-12-2021

Dr. Laxmish $\mathbf{R}$

VMMC and Safdarjung Hospital,

New Delhi, India

Dr. Vikas Gupta

VMMC and Safdarjung Hospital,

New Delhi, India

\section{Evaluation of change in the functional scoring system in children with cerebral palsy following intervention}

\author{
Dr. Laxmish R and Dr. Vikas Gupta
}

DOI: https://doi.org/10.22271/ortho.2022.v8.i1e.3034

\section{Abstract}

Cerebral palsy is a group of disorders that affect the development of movement and posture, causing activity limitation disturbances that occurred in the developing fetal or infant brain. The goal is to increase functionality, improve capabilities and sustain health in terms of locomotion, social interaction and independence. Functional scoring is done on the basis of Gross Motor Functional Classification System (Level 1 to level 5) and Functional Mobility Scale (Rating 1 to Rating 6). Study was done on 30 subjects with diagnosed Spastic Cerebral palsy less than 18 yrs. Intervention included Single Event Multi Level Surgery and Inj Botox over Calves. The study showed in the improvement of both the score following intervention and it was worth improvising the ambulatory status of a Cerebral palsy child.

Keywords: GMFCS, FMS, CP

\section{Introduction}

Cerebral palsy (CP) is a well-recognized neuro developmental condition beginning in early childhood and persisting through the lifespan. The term Cerebral Palsy was first used by William Osler in 1889 but William Little in 1862 is credited with first describing \& classifying the motor syndrome of cerebral palsy. It is defined as "a group of disorders that affect the development of movement and posture, causing activity limitation disturbances that occurred in the developing fetal or infant brain."

Cerebral palsy is caused by an insult to the developing brain. The prevalence is between 1.5 and 2.5 per 1000 live births in developed countries and spastic diplegia is the most common form. These children exhibit weakness and low endurance. Intellectual impairment occurs in about two thirds of patients with cerebral palsy. About one half of pediatric patients have seizures. Growth problems are common, as well as neurologic abnormalities such as impaired vision or hearing and abnormal touch and pain perceptions. By definition, cerebral palsy is nonprogressive; therefore, children who experience loss of previously acquired skills, or who show slowing of development, disappearance of reflexes, or unusual body odors should be evaluated for genetic, metabolic, muscular, or neuronal tumor disorders that precipitate neurodegenerative conditions.

Seventy to 80 percent of patients with cerebral palsy have spastic clinical features. Affected limbs may demonstrate increased deep tendon reflexes, tremors, muscular hypertonicity, weakness, and abnormal posture, deficit in voluntary movements and weakness in the initiation of the movement. There may be impaired fine motor function, difficulty in isolating individual movements and fatigability and a characteristic scissors gait with toe walking.

The goal of management of cerebral palsy is not to cure or to achieve normalcy but to increase functionality, improve capabilities, and sustain health in terms of locomotion, cognitive development, social interaction, and independence.

Assessment of the functional benefit from an intervention is carried out by Gross Motor Functional Classification System (GMFCS) and Functional Mobility Scale (FMS).

Gross motor functional classification system (GMFCS): The GMFCS is a standardized criterion-referenced observational evaluative measure that assesses change in gross motor function. GMFCS is a 5 level ordinal grading system based on the assessment of self-initiated movement with emphasis on function in sitting and walking. The GMFCS I children walk
Corresponding

VMMC and Safdarjung Hospital

New Delhi, India 
indoors and outdoors and climb stairs without any limitation. Children perform gross motor skills including running and jumping, but speed, balance and coordination are impaired. The GMFCS II children are limited in walking on uneven surfaces and inclines and walking in crowds or confined spaces. GMFCS III children can walk only with an assistive mobility device. They may climb stairs holding onto a railing. To take a long distance these children may need a wheelchair. GMFCS IV children may walk for a short distance on a walker. They may achieve self mobility using a power wheelchair. GMFCS V children are unable for independent mobility. All areas of motor function are limited.

Functional Mobility Scale (FMS): The FMS was developed by the group working in the Hugh Williamson Gait Laboratory of the Royal Children's Hospital, Melbourne Australia. The target population for the FMS was children with CP, GMFCS levels I to IV, aged 4 to 18 years.

One of the major aims in developing the scale was to represent the different environmental settings that are relevant to children in order to differentiate function in each. These environments include the home, school and wider community. Three items were developed and assigned distances in order to represent each environmental setting;

1. 5metres - to represent the home setting

2. 50metres - to represent the school setting

3. 500metres - to represent the community setting

The numbers were set as a guide for clinicians administering the tool, not as an exact distance.

A six point ordinal scale from 1-6 was developed depending on the amount of assistance required from the most to the least amount of assistance. The six levels were;

1. Child uses a wheelchair (or wheeled devices) for mobility

2. Child uses a walker such a posterior walker or an anterior walker

3. Child uses forearm or Canadian crutches

4. Child uses one or two single point sticks

5. Child can walk independently on level surfaces without assistive devices

6. Child can walk independently on all surfaces without assistive devices.

\section{Materials and Methods}

A prospective longitudinal study was carried out at a tertiary center at New Delhi and was conducted over a period of 2 years which included 30 patients. Subjects with diagnosed Spastic Cerebral Palsy less than 18yrs of age and can follow simple instructions are included in the study. Exclusion criteria being Children with Impaired Mobility due to other Neuro Developmental Disability like Meningomyelocele, Autism, Duchenne Muscular Dystrophy etc. and with Motor disorders like Dystonia, Athetosis, Chorea etc.

An informed written consent, a detailed clinical history, clinical examination and serial functional findings using a pre-designed proforma were performed for all the participants. Ethical clearance was obtained.

\section{Surgical Technique}

Single event multilevel surgery (SEMLS) refers to the correction of all Orthopaedic deformities in one session. It can be defined as at least two Orthopaedic procedures at different anatomical sites in each limb i.e. a minimum of four procedures. It is now considered to be the most accepted approach to correct the musculoskeletal deformities contributing to gait deviations. The frequently used procedures are muscle-tendon lengthenings, tendon transfers, rotational osteotomies and bony stabilisation procedures.

Extremity marked in the preoperative holding area. He/She brought into the operative suite and placed supine/prone depending upon the need on the operating table. The patient induced into General Endotracheal anaestheisa. IV antibiotics administered. A tourniquet placed on the patient's left/right/both proximal thigh over soft roll, and the extremity prepared and draped in the normal sterile fashion. Depending upon the requirement the following Interventions are performed.

1. SEMLS i.e Adductor longus tenotomy, Semimembranosus muscle recession, Gracilis muscle recession, Semitendinosus transfer to Adductor Magnus, Iliopsoas muscle tenotomy, Biceps femoris muscle recession, Femoral subtrochantric derotational osteotomy, Tendo Achilles lengthening depending upon the requirement.

2. Strayer procedure/vulpius procedure/Bakers procedure: where gastrocnemius recession is done.

3. Inj. Botox: given over calves depending upon the weight of the patient

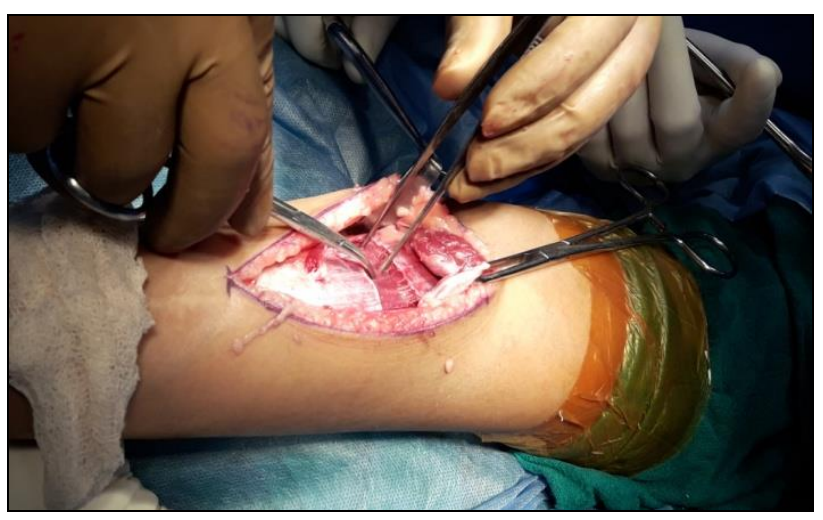

Fig 1: Strayer procedure

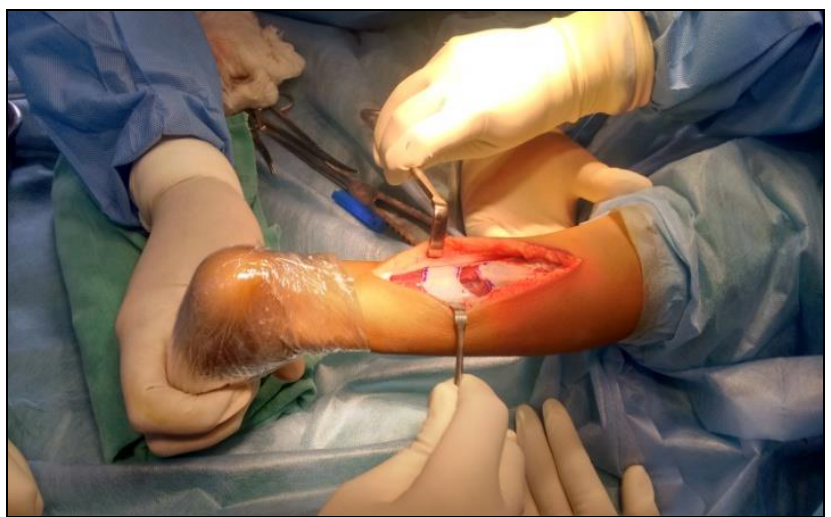

Fig 2: Vulpius procedure

Following the procedure the patient will be managed on a High groin POP (Plaster Of Paris) casting / Below knee casting depending upon the need. The patient after recovery from anaesthesia transferred to the postoperative recovery room Patient evaluated postoperatively for any cast or surgical complications.

Postoperatively patients allowed to weight bear as tolerated with/without support. The cast removed at approximately 6 weeks postoperatively and functional scoring done. The 
patients then followed at 3 months, then 6 months intervals and functional scoring done.

Functional Scoring of the child before and after Intervention will be compared to find out any improvement/ deterioration/same in the score.

\section{Result}

The present study was conducted over a period of 2 years. A total of 30 subjects including 21 male and 9 female cases fulfilling the inclusion criteria were studied. Maximun no. of subjects were in 11-15 yrs age group and minimum were in $15-20 y$ rs age group. Out of 30 cases $73.33 \%$ were Spastic Diplegics and $26.67 \%$ were Spastic Hemiplegics in study group. Out of 30 cases, Both the limbs were involved in $73.33 \%$ of cases and Left limb was involved in $6.67 \%$ of cases while Right limb was involved in $20.00 \%$ of cases in study group. Out of 30 cases, $10 \%$ of the cases underwent Inj. Botox treatment rest of the $90 \%$ of the cases underwent surgical Intervention.

\section{Changes noted in GMFCS scores following intervention Cases with Pre-op score 2}

$14(46.66 \%)$ out of 30 cases had pre op GMFCS score 2, where following intervention 11 cases $(78.57 \%)$ had same pre op score, 3 cases $(21.4 \%)$ showed improvement to score 1 with no deterioration at 6weeks interval. On follow up of 3 months, 9 cases $(64.28 \%)$ had same pre op score of 2 , \& 5 cases $(35.71 \%)$ showed score 1 . On further follow-up at 6 months interval 8 cases $(57.14 \%)$ had same pre op score and 6 cases $(42.85 \%)$ showed score 1.Therefore from GMFCS score 2, 6 cases improved to GMFCS 1, and 8 cases had same pre op score 2 till 6 months follow up with no deterioration.

\section{Cases with Pre-op score 3}

$14(46.66 \%)$ out of 30 cases had pre op GMFCS score 3, where following intervention 7 cases $(50 \%)$ had same preop score $3, \& 6$ cases $(42.85 \%)$ showed improvement to score 2 and 1 case $(7.14 \%)$ showed deterioration to score 4 at 6weeks interval. On follow up of 3 months, 7 cases $(50 \%)$ had same pre op score of $3, \& 6$ cases $(42.35 \%)$ showed score 2 and 1 case $(7.14 \%)$ showed further improvement in the score to GMFCS 1.On further follow-up at 6 months interval 4 cases $(28.57 \%)$ had same pre op score and 9 cases $(64.28 \%)$ showed score 2 and 1 case $(7.14 \%)$ showed score GMFCS 1.Therefore from GMFCS score 3, 10 cases improved to GMFCS 2, and only 4 case had same pre op score of 3 till 6 months follow up with no deterioration.

\section{Cases with Pre-op score 4}

$2(6.6 \%)$ out of 30 cases had pre op GMFCS score 4, where following intervention both the cases had same pre op score 4 at 6 weeks interval. On follow up of 3 months, 1 cases (50\%) had same pre op score of $3 \&$ the other case $(50 \%)$ showed score 3.On further follow-up at 6 months the results were same as 3 months interval. Therefore from GMFCS score 4, 1 case improved to GMFCS 3 and the other case had same same pre op score 4 without any deterioration.

\section{Changes with FMS scores following intervention Change in the FMS score at 5 mts following intervention Cases with Pre-op score 5}

$18(60 \%)$ out of 30 cases had pre op FMS score 5, where following intervention 13 cases $(72.22 \%)$ had same pre op score 5,3 cases $(16.66 \%)$ showed improvement to score 6 , and 1 case $(5.55 \%)$ each showed deterioration to score 4 and score 2 at 6weeks interval. On follow up of 3 months 12 cases $(66.66 \%)$ had same pre op score of FMS 5, 5 cases showed score 6 and 1 case $(5.55 \%)$ showed score 4 . On further follow-up at 6 months interval 12 cases $(66.66 \%)$ had same pre op score and 6 cases (33.33\%) showed score 6.Therefore cases from FMS 5, 6 cases showed improvement to score 6 and 12 cases had same pre op score 5 till 6 months interval with no deterioration.

\section{Cases with Pre-op score 4}

$10(33.33 \%)$ out of 30 cases had pre op FMS score 4, where following intervention 7 cases $(70 \%)$ had same pre op score 4 , 1 cases (10\%) showed improvement to score 5, and 2 case (20\%) showed deterioration to score 3 at 6weeks interval. On follow up of 3 months 7 cases $(70 \%)$ had same pre op score of FMS 4, 2 cases (20\%) showed score 5 and 1 case (10\%) showed score 6 . On further follow-up at 6 months interval 4 cases $(40 \%)$ had same pre op score 4,5 cases $(50 \%)$ showed score 5 and 1 case (10\%) showed score 6.Therefore cases from FMS 4, 6 cases showed improvement to score 6 and 4 cases had same pre op score 4 till 6 months interval with no deterioration.

\section{Cases with Pre-op score 2}

$2(6.66 \%)$ out of 30 cases had pre op FMS score 2, where following intervention 1 cases $(50 \%)$ had same pre op score 2 and the other case $(50 \%)$ showed improvement to score 4 at 6 weeks interval. On follow up of 3 months 1 case $(50 \%)$ had same pre op score of FMS 2 and the other case (50\%) showed score 5. On further follow-up at 6 months interval 1 case $(50 \%)$ had same pre op score and the other case $(50 \%)$ showed score 4.Therefore cases from FMS 2 showed improvement to score 5 at 3 months interval but the case deteriorated to score 4 at 6 months interval and the other case had same pre op score of 2 till 6 months interval.

\section{Change in the FMS score at 50 mts following intervention Cases with Pre-op score 5}

$13(43.33 \%)$ out of 30 cases had pre op FMS score 5, where following intervention 10 cases $(76.92 \%)$ had same pre op score 5 and 3 cases (23.07\%) showed improvement to score 6 at 6 weeks interval. On follow up of 3 months, 8 cases $(61.53 \%)$ had same pre op score of FMS 5 and 5 cases $(38.46 \%)$ showed score 6 . On further follow-up at 6 months interval 7 cases $(53.84 \%$ ) had same pre op score 5 and 6 cases $(46.15 \%)$ showed score 6.Therefore from FMS 5, 6 cases improved to score 6 , and 7 cases had same pre op score at 6 months interval with no deterioration.

\section{Cases with Pre-op score 4}

$12(40 \%)$ out of 30 cases had pre op FMS score 4, where following intervention 6 cases $(50 \%)$ had same pre op score, 4 cases $(33.33 \%)$ showed improvement to score 5 and 2 cases $(16.66 \%)$ showed deterioration to score 2 at 6weeks interval. On follow up of 3 months, 6 cases $(50 \%)$ had same pre op score of FMS 4, 4 cases $(33.33 \%)$ showed score 5,1 case $(8.33 \%)$ showed score 6 and 1 case $(8.33 \%)$ showed score 2. On further follow-up at 6 months interval 6 cases $(50 \%)$ had same pre op score, 5 cases $(41.66 \%)$ showed score 5 and 1 case $(8.33 \%)$ showed score 6.Therefore from FMS 4, 5 cases improved to FMS 5, 1 case showed improvement to score 6 and 6 cases had same pre op score 4 till 6 months interval with no deterioration. 


\section{Cases with Pre-op score 3}

$4(13.33 \%)$ out of 30 cases had pre op FMS score 3, where following intervention 3 cases $(75 \%)$ had same pre op score and 1 cases $(25 \%)$ showed improvement to score 4 at 6 weeks interval. On follow up of 3 months results were same. On further follow-up at 6 months interval 1 case (25\%) had same pre op score and 3 cases $(75 \%)$ showed score 4 . Therefore from FMS 3, 3 cases improved to score 4 and only 1 case had same pre op score 3 till 6 months interval with no deterioration.

\section{Cases with Pre-op score 2}

Only 1 case $(6.66 \%)$ out of 30 cases had pre op FMS score 2, where following intervention it showed deterioration to score FMS 1 at both 6 weeks and 3 months interval. On follow up of 6 months interval it changed to its pre op score 2. So from FMS 2 the case deteriorated till 3 months and returned to its pre op score 2 at 6 months interval. Therefore case with Pre op score 2 showed no improvement.

\section{Change in the FMS score at 500 mts following intervention. \\ Cases with Pre-op score 5}

$12(40 \%)$ out of 30 cases had pre op FMS score 5, where following intervention 9 cases $(75 \%)$ had same pre op score 5 and 3 cases (25\%) showed improvement to score 6 at 6weeks interval. On follow up of 3 months, 7 cases $(58.33 \%)$ had same pre op score of FMS 5 and 5 cases (41.66\%) showed score 6. On further follow-up at 6 months interval 6 cases (50\%) had same pre op score 5 and 6 cases (50\%) showed score 6.Therefore from FMS 5, 6 cases improved to score 6 gradually, and the other 6 cases had same pre op score at 6 months interval with no deterioration.

\section{Cases with Pre-op score 4}

$12(40 \%)$ out of 30 cases had pre op FMS score 4, where following intervention 9 cases $(75 \%)$ had same pre op score 4 , 1 case $(8.33 \%)$ showed improvement to score 5 and 2 cases $(16.66 \%)$ showed deterioration to score 2 at 6 weeks interval. On follow up of 3 months, 6 cases $(50 \%)$ had same pre op score of FMS 4, 4 cases $(33.33 \%)$ showed score 5,1 case $(8.33 \%)$ showed score 6 and 1 case $(8.33 \%)$ showed score 2. On further follow-up at 6 months interval 7 cases $(58.33 \%)$ had same pre op score, 4 cases $(33.33 \%)$ showed score 5 and 1 case $(8.33 \%)$ showed score 6.Therefore from FMS 4, 4 cases improved to FMS 5, 1 case showed improvement to score 6 and 7 cases had same pre op score 4 till 6 months interval with no deterioration.

\section{Cases with Pre-op score 3}

$4(13.33 \%)$ out of 30 cases had pre op FMS score 3 , where following intervention 3 cases $(75 \%)$ had same pre op score and 1 cases $(25 \%)$ showed improvement to score 4 at 6weeks interval. On follow up of 3 months results were same. On further follow-up at 6 months interval 2 case $(50 \%)$ had same pre op score and 2 cases $(50 \%)$ showed score 4 .Therefore from FMS 3, 2 cases improved to score 4 and the other 2 case had same pre op score 3 till 6 months interval with no deterioration.

\section{Cases with Pre-op score 2}

2 cases $(6.66 \%)$ out of 30 cases had pre op FMS score 2, where following intervention 1 case $(50 \%)$ improved to score FMS 4 from 6 weeks till 6 months follow up and the other case $(50 \%)$ showed score of 1 at 6 weeks and 3 months interval. On follow up of 6 months interval it changed to its pre op score 2. So from FMS 2, 1 of the case had same pre op score and the other case improved to score 4.

\section{Discussion}

Cerebral palsy (CP) is a well-recognized neuro developmental condition beginning in early childhood and persisting through the lifespan. Mobility involves "changing location of transferring from one place to another". It stands to reason that optimal mobility of children with $\mathrm{CP}$ will encourage their participation with their peers and family. As already mentioned the goal of management of cerebral palsy is not to cure or to achieve normalcy but to increase functionality, improve capabilities, and sustain health in terms of locomotion, cognitive development, social interaction, and independence.

Our study included two classification systems to assess the ambulatory status in a CP child i.e Gross Motor Functional Classification System (GMFCS) and Functional Mobility scale (FMS). Interventions in our study included Surgery and Inj.Botox.

\section{Age distribution}

Adrienne Harvey et al in 2007 conducted a study to examine the ability of the Functional Mobility Scale (FMS) to detect change in children with cerebral palsy $(\mathrm{CP})$ undergoing single event multilevel surgery (SEMLS) which included 66 children with mean age of $10 \mathrm{yrs}$.

Pam Thomason et al in 2012 conducted a study on Single Event Multilevel Surgery in children with bilateral spastic cerebral palsy. Which included 19 children with mean age of 9.7 yrs.

In our study, the minimum and maximum age of study group was 3 years and 20 years respectively with mean age of 10.37 \pm 4.43 years.

\section{Sex distribution}

In our study there were $21(70 \%)$ males and $9(30 \%)$ females which was in agreement with study conducted by Adrienne Harvey et al in which 34 were males and 32 were females patients. Similar sex distribution founded in Christian Schranza et al in which 9 were males and 5 were females.

However there is no correlation between our study results and sex distribution. Male preponderance can be explained by apathy towards girl child in our society.

\section{Side Involvement}

Our study founded $73.33 \%$ of the cases had bilateral involvement, $20 \%$ had right sided involvement and $6.66 \%$ had left sided involvement.

\section{Changes In The Scores}

\section{GMFCS score changes following intervention}

Score 1: Intervention not required.

Score 2: In our study, cases with score 2 shows improvement to score 1 without any deterioration.

Score 3: In our study, cases with score 3 shows improvement to score 1 without any deterioration.

Score 4: In our study, cases with score 4 shows improvement till score 3 without any deterioration.

Score 5: In our study, there were no cases with GMFCS score 5.

Overall $56.67 \%$ of the cases showed improvement in GMFCS score and $43.3 \%$ of cases showed same pre op score with no deterioration. 
In Francesco et al, study on CP child with GMFCS 4 following surgery showed no improvement in the score.

\section{FMS score changes following intervention}

FMS Score Changes following Intervention at 5 Mts

Score 6: Intervention not required.

Score 5: In our study, cases with score 5 shows improvement to score 6 without any deterioration.

Score 4: In our study, shows improvement to score 6 without any deterioration.

Score 3: In our study, there were no cases with FMS score 3.

Score 2: In our study, cases with score 2 shows no improvement.

Score 1: In our study, there were no cases with FMS score 1. Overall $43.3 \%$ of the cases showed improvement in FMS score at $5 \mathrm{mts}$ and $56.7 \%$ of the case had same pre op score with no deterioration in the score.

\section{FMS Score Changes following Intervention at 50 Mts}

Score 6: Intervention not required.

Score 5: In our study, cases with score 5 shows improvement to score 6 without any deterioration.

Score 4: In our study, cases with score 4 shows improvement to score 6 without any deterioration.

Score 3: In our study, cases with score 3 shows improvement to score 4 without any deterioration.

Score 2: In our study, $6.66 \%$ of the cases had score 2, amongst which all of them had same score 2 till 6 month interval. This shows that cases with score 2 shows no improvement.

Score 1: In our study, there were no cases with FMS score 1. Overall $50 \%$ of the cases showed improvement in FMS score at $50 \mathrm{mts}$ and the other $50 \%$ of the cases had same pre op score with no deterioration in the score.

\section{FMS Score Changes following Intervention at 500 Mts.}

Score 6: Intervention not required.

Score 5: In our study, cases with score 5 shows improvement to score 6 without any deterioration.

Score 4: In our study, cases with score 4 shows improvement to score 6 without any deterioration.

Score 3: In our study, cases with score 3 shows improvement to score 4 without any deterioration.

Score 2: In our study, with score 2 can be improved to score 4.

Score 1: In our study, there were no cases with FMS score 1. Overall $50 \%$ of the cases showed improvement in FMS score at $500 \mathrm{mts}$ and the other $50 \%$ of the cases had same pre op score with no deterioration in the score.

In general all the cases were used to show change for the group as a whole. More children who had intervention, particularly at the first time point post operatively showed same functional scores .By the third post operative time point, more number of cases showed improvement than deterioration.

\section{Conclusion}

- The GMFCS and FMS scores were able to detect changes in the mobility in children with $\mathrm{CP}$ as defined by one level of improvement or deterioration.

- This study provides some evidence of the ability of the functional scores to detect clinically important change.

- The FMS was developed to provide an activity outcome measure for children with CP specific to mobility.

- It is concluded that the intervention is worth in improvising the ambulatory status of a $\mathrm{CP}$ child especially GMFCS 2 and 3.

- SEMLS has a beneficial effect on ambulatory status of a CP child.

\section{References}

1. Rosenbaum P. The definition and classification of cerebral palsy. J Pediatr Orthop. 2007;49(6):480.

2. Graham HK. Classifying cerebral palsy. J Pediatr Orthop. 2005;25:127-128.

3. Bache CE, Selber P, Graham HK. The management of spastic diplegia. Current Orthopaedics 17:88-104.

4. Abel MF, Damiano DL, Pannunzio M, Bush J. Muscletendon surgery in diplegic cerebral palsy functional and mechanical changes. J Pediatr Orthop 19:366-375.

5. Baker R, Jasinski M, Maciag-Tymecka I, MichalowskaMrozek J, Bonikowski M, Carr L et al. Botulinum toxin treatment of spasticity in diplegic cerebral palsy. Develp Med and Child Neuro 44:666-675.

6. Browne AO, McManus F. One-session surgery for bilateral correction of lower limb deformities in spastic diplegia. J Pediatr Orthop 1987;7:259-261.

7. Beckung E, Hagberg G. Correlation between ICIDH handicap code and Gross Motor Function Classification System in children with cerebral palsy. Develp Med and Child Neuro 42:669-673.

8. Bjornson KF, Graubert CS, et al. Validity of the gross motor function measure. Pediatric Physical Therapy 10:43-47.

9. Bjornson KF, Graubert CS, et al. Test-retest reliability of the Gross Motor Function Measure in children with cerebral palsy. Physical and Occupational Therapy in Pediatrics 18:51-61.

10. Silver CM, Simon SD. Gastrocnemius-muscle recession (Silfverskiold operation) for spastic equinus deformity in cerebral palsy. J Bone Joint Surg A 1959;41:1021-28.

11. Boyd RN, Hays RM. Outcome measurement of effectiveness of botulinum toxin type A in children with cerebral palsy: an ICIDH-2 approach. European $\mathbf{J}$ of Neuro 8(5):167-177.

12. Palisano R, Rosenbaum P, Walter S, Russell D, Wood E. Development and reliability of a system to classify gross motor function in children with cerebral palsy. Develp Med and Child Neuro. 39:214-223.

13. Graham HK, Harvey A, Rodda, Nattrass GR, Pirpiris M. The Functional Mobility Scale. J Pediatr Orthop 24(5):514-520. 